\title{
Three Dimensional Optical Microstructure Analysis of Ferrite and Pearlite Phases in a Medium Carbon Steel
}

\author{
Bryan Turner ${ }^{1}$, Satya Ganti ${ }^{1}$, Veeraraghavan Sundar $^{1}$ and Bill Davis ${ }^{2}$ \\ 1. UES Inc., Robo-Met.3D Services, Dayton, OH \\ 2. WSD Consulting, Ciincinnati, $\mathrm{OH}$
}

This work presents a three dimensional microstructural analysis of medium carbon steels, based on serial sectioning using optical microscopy. The microstructural phases considered here are ferrite and pearlite. Typically conventional 2D microscopy is used to analyze these phases, with eddy current or ultrasonic methods as alternatives. Medium carbon steel samples were heat treated in a furnace and either slowly cooled in the furnace, or cooled at higher rates. This resulted in different pearlitic microstructures in the samples. The analysis was conducted with Robo-Met.3D, a fully automated serial sectioning system that generates two-dimensional optical microstructural data for three dimensional reconstructions. Based on optical microscopy, volume cubes of hundreds of microns on edge were imaged and reconstructed to conduct grain size and phase volume analysis. The system proved capable of collecting image data with high fidelity, and controlled slice thickness in an automated manner. The resultant dataset was useful in visualization and analysis of pearlite and ferrite phases in steel samples.

An off-the-shelf, un-heat treated, cold drawn, 1045 medium carbon steel was chosen for a demonstration specimen as it is a common material, very well studied, is straightforward to polish, responds well with simple etchants. The sample used here was sourced commercially and mounted conventionally for metallography.

Robo-Met.3D ${ }^{\circledR}$ is a fully automated serial sectioning system that generates two dimensional optical microstructural data for three dimensional reconstructions and analysis. Common applications of RoboMet.3D include studying additively manufactured components ${ }^{\mathrm{i}}$, analysis thermal barrier coatings ${ }^{\mathrm{ii}}$, and fiber orientation effects in ceramic matrix composites ${ }^{\mathrm{iii}}$.

The microstructure of 1045 steel typically features mean grain diameters between 16 to $32 \mu$ per ASTM A108. A mosaic, or montage of $2 \times 2$ tiles of 500x images was collated, with a resolution of $0.21 \mu$ along the $\mathrm{X}$ and $\mathrm{Y}$ axes. A total of 50 slices, or sections were collected for a $\mathrm{z}$-height of $92 \mu$. An average slice thickness of $1.8 \mu$ was maintained consistently through this collection. Samples were etched with $2 \%$ Nital for contrast. All samples were imaged using this protocol.

The resultant image pixel size $451.05 \mu$ along $\mathrm{X}$ and $281.51 \mu$ along $\mathrm{Y}$, after stitching, aligning and cropping to exclude unwanted regions. For 3D analysis, the raw $2 \mathrm{D}$ images were stitched and aligned for accurate post-processing. The loaded z-stack of 50 slices was processed with subsampling along Y direction and full resolution along $\mathrm{X}$ and $\mathrm{Z}$ directions. A 3D isosurface was created without any filtering to extract the volume distribution of the two phases. Thresholding was performed on pixel values as for the 2D analyses (Figs. 1,2). A combination of Fiji and ImagePro software suites was used in this analysis. 


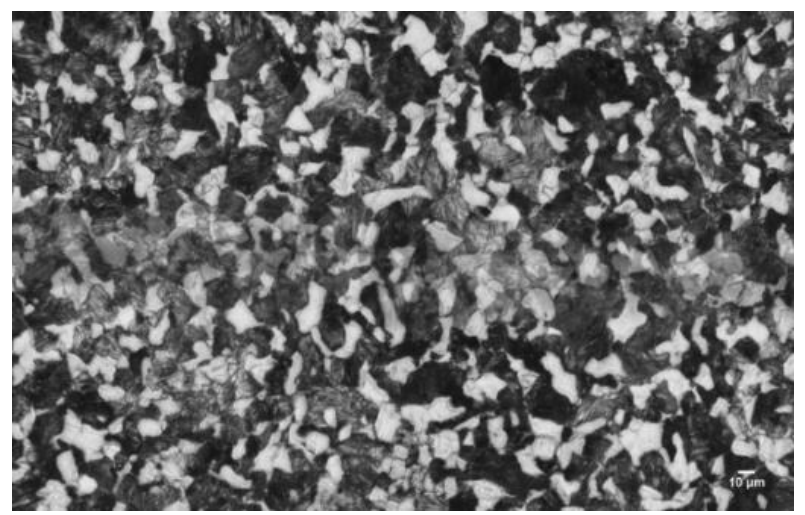

Figure 1. Raw 2D Image (Etched with 2\% Nital)

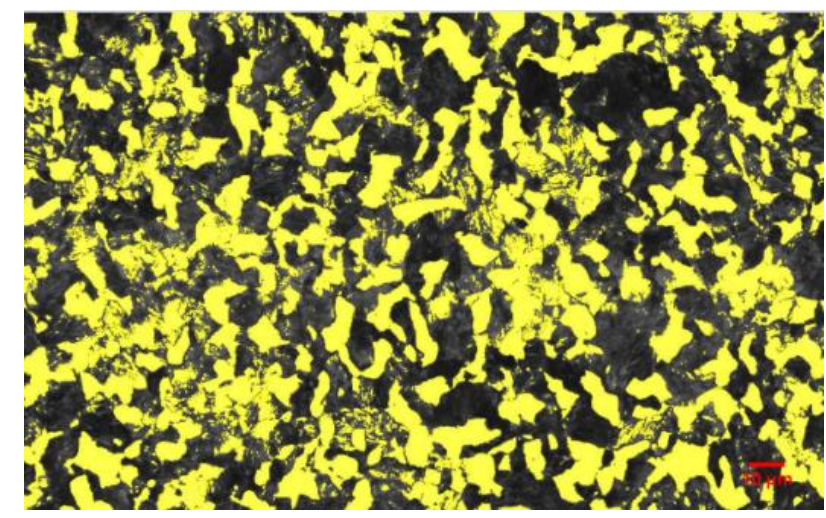

Figure 2. Segmented 2D Image with Pearlite in Yellow

The 2D and 3D image sets (Figs. 1-3) were analyzed for the relative fraction of pearlite and ferrite phases. The 2D area analyses yielded a Ferrite content of $64 \%$ and Pearlite content of $36 \%$. Volumetric 3D analyses yielded a Ferrite content of $71 \%$ and Pearlite content of $29 \%$. The 3D data may be considered more representative as it minimizes section-dependent variation ${ }^{\mathrm{iv}}$.

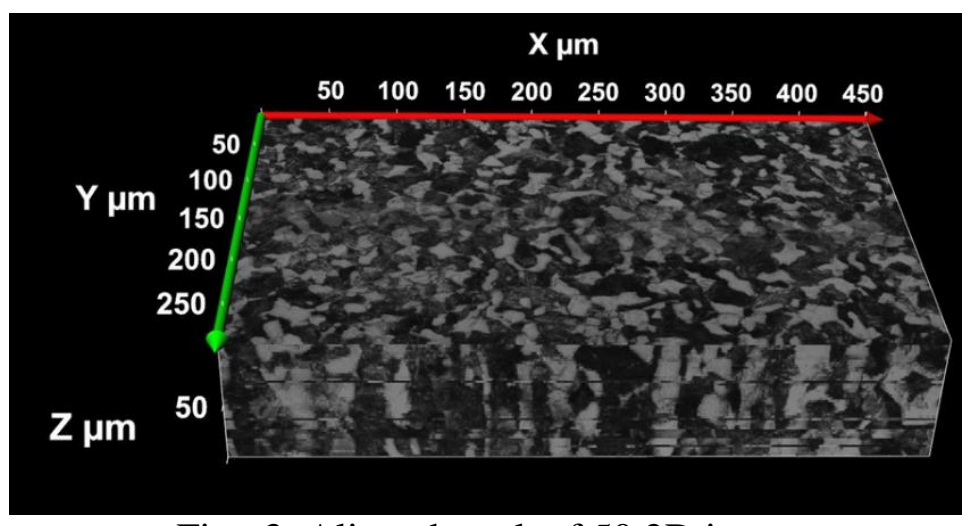

Fig. 3: Aligned stack of 50 2D images.

The study successfully demonstrates the use of RoboMet.3D ${ }^{\circledR}$. The system proved capable of collecting image data with high fidelity, and controlled slice thickness in an automated manner. The resultant dataset was useful in visualization and analysis of pearlite and ferrite phases in 1045 steel samples.

\section{References:}

[1] Seifi, Mohsen et al, Proc. of World Conference on Titanium, 13th. Warrendale, PA/Hoboken, NJ: TMS/Wiley (2016).

[2] Brake, M. R. W., Aaron Christopher Hall, and Jonathan D. Madison, Surface and Coatings Technology 310 (2017), p. 70.

[3] Bricker, Stephen, et al, SPIE/IS\&T Electronic Imaging. International Society for Optics and Photonics (2015).

[4] Ganti, S. et al, Practical Metallography 54.2 (2017), p. 77. 